\title{
Linear Digital Cancellation with Reduced Computational Complexity for Full-Duplex Radios
}

\author{
Muhammad Sohaib Amjad, Ozgur Gurbuz \\ Faculty of Engineering and Natural Sciences (FENS) \\ Sabanci University \\ Istanbul, Turkey \\ muhammadsohaib,ogurbuz@ sabanciuniv.edu
}

\begin{abstract}
Recent works have demonstrated the feasibility and potential of full-duplex (FD) wireless systems to double the spectral efficiency of half-duplex (HD) systems. Self-interference (SI) cancellation is the key to FD communication and the residual SI is the major factor determining the performance of an FD radio. This paper presents a novel frequency domain based approach, for the reconstruction of SI signal in digital domain. Unlike the existing time domain reconstruction approach, which requires convolution, the proposed approach is simple and uses FFT processing for SI signal reconstruction. By means of computational complexity evaluation, it is shown that the proposed reconstruction approach offers $61 \%$ reduction in the floating point operations required to reconstruct SI signal. Furthermore, via detailed simulations on the performance of digital SI cancellation under fading channels, it is demonstrated that the SI suppression achieved with the proposed approach is not only comparable to existing approach, but with frequency domain SI channel estimates, it can offer $5-7 \mathrm{~dB}$ better cancellation under highly frequency selective fading conditions, suggesting its suitability for long range transmission.
\end{abstract}

Keywords-Digital Cancellation, Self-Interference, Frequency Domain Reconstruction, Time Dispersive Fading.

\section{INTRODUCTION}

Present wireless system require separate resources in time or frequency for reliable communication. As a result, all current wireless devices operate in half-duplex mode, where separate resources are allocated for transmission and reception. FD is a promising wireless technology with the ability and potential to reduce the growing wireless spectral congestion. An FD radio, only requires a single resource to transmit and receive simultaneously, which theoretically cuts the spectrum requirement by half, resulting in a twofold increase in the spectral efficiency as compared to an HD system, or an accommodation of twice the number of users in the same cell zone.

The fundamental challenge in the realization of FD wireless communication, is the huge power difference between the SI signal produced as a result of radio's own transmission, and the desired signal arriving from a distant transmitting antenna. The SI signal, due to its close proximity to the receiver, more or less occupies the whole dynamic range of the data converters in the received signal processing path, making the processing of the desired signal impossible.

To prevent the saturation of the data converter's dynamic range, a considerable amount of SI suppression is required

This work was supported in part by TUBITAK grant no. 215E326 first in the analog domain, i.e. at the RF stage. The remaining SI signal, is then suppressed in the digital domain. Any residual SI after digital domain cancellation ultimately acts as (additive) noise, and decreases the signal-to-noise ratio (SNR) of the desired signal, which eventually reduces the system throughput. Therefore, operation of FD communication at maximum capacity, requires a full suppression of the SI signal to the receiver's noise floor.

Recent works [1]-[11] have presented different system architecture and SI mitigation techniques for enabling FD communication. Apart from SI suppression techniques employed in analog/RF stage, implementation of an FD transmission on an existing HD system, such as, IEEE $802.11 \mathrm{a} / \mathrm{g}$ standard for WiFi radios, requires some major structural modification of the design flow in digital domain. This primarily includes the reconstruction of an approximate SI signal in baseband with the aid of the SI channel estimate, and then its subtraction from the total received signal, which is required to be done prior to receiver's processing of the desired signal.

Digital self-interference cancellation plays a key role in determining the performance of an FD system, as it primarily quantifies the SNR levels after the suppression of the SI. To achieve linear digital cancellation, previous designs have used the time domain approach for SI signal reconstruction with least square channel estimates acquired either in time domain [1], [12] or frequency domain [2], [9]. However, the performance of time domain reconstruction with time domain estimates [1], [12] suffers significantly in channels with prolonged impulse response because of the limited length of the cyclic prefix (CP). Likewise, time domain reconstruction with frequency domain estimates [2], [9] offers poorer digital cancellation, because taking the inverse fast Fourier transform (IFFT) of the frequency domain estimate distributes the concentrated channel taps power over FFT size. Additionally, time domain reconstruction technique requires convolution operation, whose computationally complexity can grow as $O\left(N^{2}\right)$.

This work proposes a novel frequency domain based approach for the reconstruction of SI signal. The proposed approach uses FFT processing, which is computationally less demanding than convolution (used in time domain reconstruction), as shown in the comparative computational complexity analysis in this paper. Additionally, we have conducted a detailed performance analysis and comparison of the proposed frequency domain approach and the existing time domain approach, with the help of a baseband system model for an FD OFDM system, considering the widespread IEEE 802.11a/g 


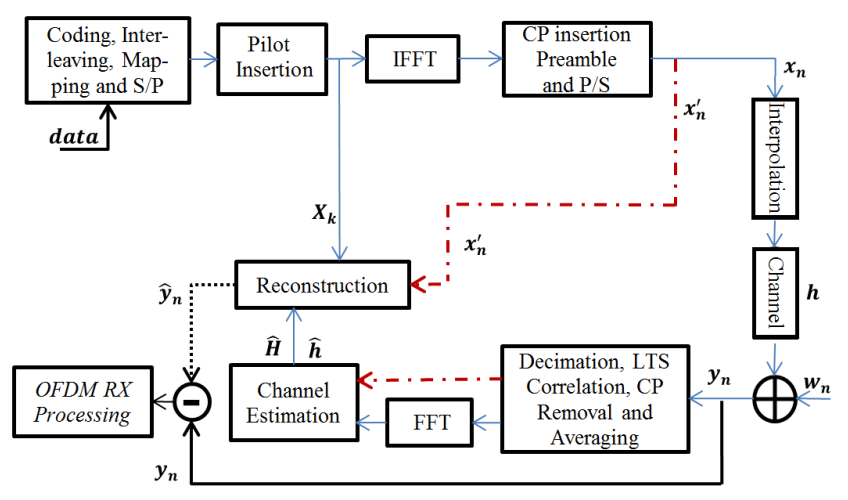

Figure 1. Baseband model of FD OFDM system.

standard, and modeling a multi path fading environment with different types and levels of fading. It is shown that the proposed frequency domain reconstruction approach is nearly three times more computationally efficient, and it demonstrates $5-7 \mathrm{~dB}$ improved digital cancellation under highly selective fading conditions.

The rest of the article is organized as follows. Section II describes the system model. The methods for the suppression of SI signal in digital domain are explained in Section III. In section IV, the computational complexities of different SI signal regeneration techniques are evaluated. Section V presents the performance results of the compared digital cancellation approaches, and section VI concludes this article.

\section{SySTEM MODEL}

Fig. 1 presents the structure of our baseband FD node. The model is based on IEEE 802.11a/g standard HD OFDM system. A list of key parameters of IEEE 802.11a/g standard that are included in our model is presented in Table I. Besides the systematic OFDM blocks, the noteworthy units in our FD model are the additional blocks of channel estimation and reconstruction, which are required prior to receiver's processing of the desired signal. At the receiver of FD radio, the baseband received signal $y_{n}$ can be expressed as

$$
y_{n}=h * x_{n}+r_{n}+w_{n},
$$

where $x_{n}$ is the SI signal, $h$ is the channel impulse response (CIR) corrupting $x_{n}, r_{n}$ represents the desired signal and $w_{n}$ is AWGN noise. It is assumed that during the channel estimation process, no signal other than SI signal, is at hand (for best possible estimation of the SI channel, $h$ ), i.e. $r_{n}=0$, thus reducing (1) to

$$
y_{n}=h * x_{n}+w_{n}
$$

Table I. KEY PARAMETERS OF OUR BASEBAND FD MODEL

\begin{tabular}{l|l}
\hline \hline Modulation & BPSK, QPSK, 16-QAM \\
\hline No of Subcarriers & 52 \\
\hline No of Pilots & 4 \\
\hline OFDM Symbol duration & $4 \mu \mathrm{s}$ \\
\hline Guard Interval & $800 \mathrm{~ns}$ \\
\hline Cyclic Prefix (CP) length & $16 \mathrm{Samples}$ \\
\hline Signal Bandwidth & $16.66 \mathrm{MHz}$ \\
\hline Subcarrier Spacing & $312.5 \mathrm{kHz}$ \\
\hline FFT Size & 64 \\
\hline
\end{tabular}

Considering the OFDM based air interface, of IEEE $802.11 \mathrm{a} / \mathrm{g}$, the preamble length is taken as 12 symbols with the first 10 belonging to the short training sequence (STS) required for synchronization, packet detection and carrier offset correction, and the remaining two symbols belong to the long training sequence (LTS) used to compute channel state information and symbol synchronization, where each LTS symbol contains identical training sequence.

\section{Digital Self-Interference CANCELlation}

Cancellation of SI in digital domain is a two step process. First, the SI channel is estimated using the LTS symbols packed in the preamble of an OFDM burst. Afterwards, the obtained channel estimate is used to equalize the known transmitted information, so that the reconstructed signal innate the same channel properties as that imposed on the received SI signal.

\section{A. Estimation of Self-Interference Channel}

Channel estimation is the task of estimating the gain of SI channel that essentially disrupts an FD radio's own transmissions, before it actually reaches the receiver. To efficiently complete this task in wireless systems, identical OFDM symbols carrying training sequence (LTS) for each carrier are used, where averaging of these received LTS symbols is used to enhance the quality of the channel estimate.

1) Least Square Time Domain Estimation: Least square time domain estimation (LS-TDE) approach is used in [1], [6], [12] for SI channel estimation in FD systems. In LS-TDE, CIR is computed by using the averaged receive LTS symbols. From (2), the received time domain $l$ LTS symbols having $n$ samples per symbol are given as

$$
y_{l, n}=h * x_{n_{L T S}}+w_{l, n} .
$$

The time domain convolution can be expressed as a matrix vector multiplication, specifically for preamble, since it is known and pre-defined. Thus the above channel equation can be written as

$$
y_{l, n}=\mathbf{X}_{\mathbf{n}_{\mathbf{L T S}}} h+w_{l, n},
$$

where $h$ is CIR vector and $\mathbf{X}_{\mathbf{n}_{\text {LTS }}}$ is the Toeplitz matrix formed using the known LTS as shown below:

$$
\mathbf{X}_{\mathbf{n}_{\mathbf{L T S}}}=\left[\begin{array}{ccccc}
x_{1} & x_{n} & x_{n-1} & \cdots & x_{n-L+2} \\
x_{2} & x_{1} & x_{n} & \cdots & x_{n-L+3} \\
\vdots & \vdots & \vdots & \ddots & \vdots \\
x_{n-1} & x_{n-2} & x_{n-3} & \cdots & x_{n-L} \\
x_{n} & x_{n-1} & x_{n-2} & \cdots & x_{n-L+1}
\end{array}\right] h=\left[\begin{array}{c}
h_{1} \\
h_{2} \\
\vdots \\
h_{L-1} \\
h_{L}
\end{array}\right] .
$$

Here, the parameter $L$ states the maximum length of the CIR that can be estimated and $\mathbf{X}_{\mathbf{n}_{\text {LTS }}}$ is a circular matrix of order $n \times L$. The matrix $\mathbf{X}_{\mathbf{n}_{\mathrm{LTS}}}$ can be pre-computed since we know the values of transmitted LTS symbols in advance. The CIR estimate is thus calculated as given in [13]

$$
\hat{h}=\mathbf{X}_{\mathbf{n}_{\mathbf{L T S}}}^{\dagger} y_{l, n} \text {. }
$$

Where $\mathbf{X}_{\mathbf{n}_{\mathbf{L T S}}}^{\dagger}$ denotes Moore-Penrose (pseudo) inverse of $\mathbf{X}_{\mathbf{n}_{\text {LTS }}}$ and $y_{l, n}$ is the average of $l$ LTS symbols. The channel 
frequency response estimate can then be obtained by performing the FFT of the acquired impulse response as

$$
\hat{H}_{\mathrm{LS}-\mathrm{T}}=\mathrm{FFT}_{k}\left\{\hat{h}_{n}\right\} .
$$

2) Least Square Frequency Domain Estimation: Least square frequency domain estimation (LS-FDE) technique uses the averaged receive LTS symbols obtained after FFT processing, to compute the SI channel estimate. The $l$ received LTS symbols $Y_{l, k}$ for $k$ carriers are obtained as the inner product of the training sequence symbols vector $X_{k_{L T S}}$ and the channel frequency response $H_{k}$ plus additive noise $W_{l, k}$ as

$$
Y_{l, k}=H_{k} \cdot X_{k_{L T S}}+W_{l, k},
$$

where $X_{k_{L T S}}$ is a vector containing the training sequence. The channel estimate $\hat{H}_{k}$ is computed as given in [13];

$$
\hat{H}_{k}=Y_{l, k} \cdot / X_{k_{L T S}}
$$

The time domain CIR can then be evaluated as

$$
\hat{h}_{\mathrm{LS}-\mathrm{F}}=\operatorname{IFFT}_{n}\left\{\hat{H}_{k}\right\} .
$$

\section{B. Reconstruction of Self-Interference Signal}

The procedure of SI signal reconstruction is similar to that of equalization, in-fact it is equivalent to reverse of equalization. To incorporate the channel effects on the reconstructed SI signal, the obtained SI channel estimate is processed with the known transmitted data as explained next.

1) Time Domain Reconstruction: The time domain reconstruction (TD-R) employed in [1], [2], [6], [9], simply applies the convolution operation on $x_{n}^{\prime}$ (the time domain TX samples prior to interpolation filter as shown in Fig.1) and $\hat{h}$ (the CIR estimate) obtained using (5) or (9), with the resultant signal given as

$$
y_{n}^{\prime}=\hat{h} * x_{n}^{\prime} .
$$

Following the convolution operation the output $y_{n}^{\prime}$ is interpolated to obtain the reconstructed signal $y_{n}$ as presented in Fig. 2 with red dashed lines.

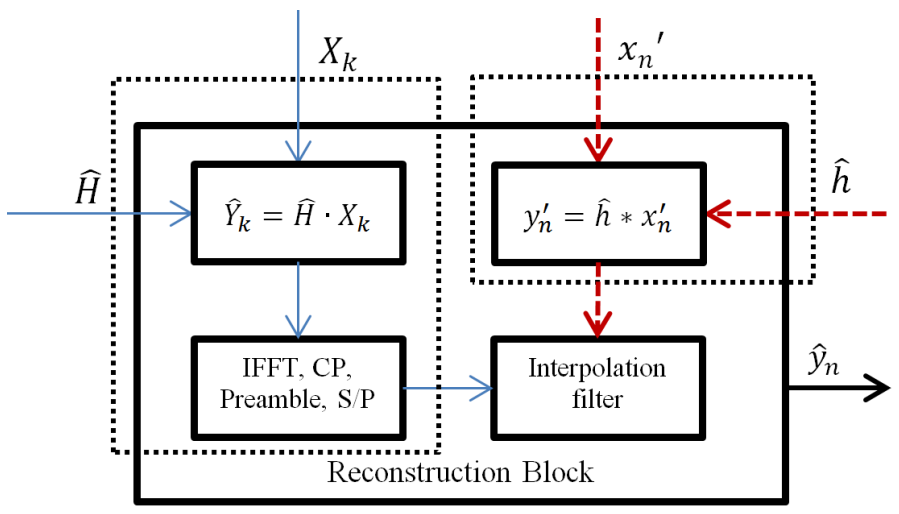

Figure 2. Structure implementing the frequency domain approach for the reconstruction of self-interference signal in digital domain.
2) Frequency Domain Reconstruction: The proposed frequency domain reconstruction (FD-R) technique takes in the baseband symbols $X_{k}$ (obtained after pilot insertion) and equalizes them with the frequency domain channel estimate $\hat{H}_{k}$ obtained using (6) or (8). The reconstructed signal is obtained as follows:

$$
\hat{Y}_{N, k}=\hat{H}_{N, k} \cdot X_{N, k} \quad \rightarrow \quad y_{n}^{\prime}=\operatorname{IFFT}_{N, k}\left\{\hat{Y}_{N, k}\right\},
$$

where $N$ represents the number of transmitted symbols, $k$ is the FFT size and $\hat{Y}_{N, k}$ are the reconstructed $N$ OFDM symbols. Note that, the same channel estimate $\hat{H}$, needs to be repeated $N$ times, so that each transmitted symbol $X_{k}$ can be equalized. Once the symbols are equalized, they go through the same process of IFFT, CP insertion, parallel to serial conversion, preamble attachment and finally through interpolation filter to obtain time domain reconstructed SI signal $\hat{y}_{n}$ as shown in Fig. 2 with blue dashed lines.

\section{Computational Complexities of Si Signal REGENERATION}

Computational complexity is calculated as the number of floating point operations (flops) required to compute an instance or input of a given algorithm. In this section, we analyze and compare the implementation requirements in terms of computational complexity of different SI signal regeneration processes, which comprise of SI channel estimation and SI signal reconstruction stages.

\section{A. Estimation Complexity}

Computational requirement of the two discussed SI channel estimation techniques vary because of their implementation structures as analyzed next.

1) Time Domain Estimate: The LS-TDE is done prior to FFT processing, and from (5) it can be deduced that it requires $(n \cdot L)$ complex multiplications and $(n \cdot L)$ complex additions to compute this estimate. Here, $n$ represents the length of the averaged receive LTS samples $y_{l, n}$, and $L$ represents the length of the CIR. A single complex multiplication requires four real multiplications and two real additions, and a single complex addition requires two real additions [14]. Thus, the computational requirement of LS-TDE is obtained as

$$
\begin{array}{cr}
4(n \cdot L) & \text { real multiplications, } \\
4(n \cdot L) & \text { real additions. }
\end{array}
$$

Note that, using this estimate with proposed FD-R approach, requires the estimate to be transformed into frequency domain, which increases the flop count.

2) Frequency Domain Estimate: From (8) it can be seen that the LS-FDE only requires a single FFT processing to compute the channel estimate. A single $k$ points Radix-2 FFT processing requires $(k / 2) \cdot \log _{2}(k)$ complex multiplications and $(k) \cdot \log _{2}(k)$ complex additions as given in [14]. Therefore, the computational complexity of this $k$ points Radix-2 FFT is computed as given in [14], [15]

$$
\begin{array}{lr}
2 k \cdot \log _{2}(k)-7 k+12 & \text { real multiplications, } \\
3 k \cdot \log _{2}(k)-3 k+4 & \text { real additions, }
\end{array}
$$

where $k$ is FFT size and it is required to be greater than one. When this estimate is used prior to TD-R, an additional IFFT processing is required, which doubles the flop count. 


\section{B. Reconstruction Complexity}

The implementation structures of the two reconstruction approaches discussed earlier are different mainly due to their separate domains of operation.

1) Time Domain Reconstruction: The TD-R approach requires convolution operation, which can grow computationally expensive with transmitted packet size. The implementation of convolution operation is achieved in two basic ways: (1) Discrete Convolution (DC), and (2) Circular Convolution (CC). The DC simply applies the multiply and accumulate (MAC) operation. Thus, to reconstruct $N$ OFDM symbols containing $(n+C P)$ samples, each using $L$ channel coefficients requires

$$
\begin{array}{cc}
(4(n+C P) \cdot L) \cdot N & \text { real multiplications, } \\
(4(n+C P) \cdot L) \cdot N & \text { real additions. }
\end{array}
$$

To avoid the expensive MAC operation utilized by DC, the circular convolution is usually used, which uses the FFT processing to lower the computational requirements. The CC algorithm, first takes the $n$ point FFT of both the OFDM symbol and the channel coefficients (where $n$ represents the number of samples in one OFDM symbol), then multiplies them in frequency domain and converts the equalized result back into time domain via IFFT process. Thus, the computational requirements using (14) and (15) for Radix-2 FFT are given as

$$
\begin{array}{cc}
\left(6 n \cdot \log _{2}(n)-17 n+36\right) \cdot N & \text { real multiplications } \\
\left(9 n \cdot \log _{2}(n)-7 n+12\right) \cdot N & \text { real additions. }
\end{array}
$$

2) Frequency Domain Reconstruction: The proposed FDR approach takes the $N$ known transmitted OFDM symbols, each containing $k$ data symbols, equalizes them with the acquired frequency domain channel estimate via multiplication operation, and converts the resultant symbols into time domain through IFFT processing. The computational requirements for FD-R using (14) and (15) are thus evaluated as

$$
\begin{array}{cc}
\left(2 k \cdot \log _{2}(k)-3 k+12\right) \cdot N & \text { real multiplications, } \\
\left(3 k \cdot \log _{2}(k)-k+4\right) \cdot N & \text { real additions. }
\end{array}
$$

Table II presents the computational complexities of different estimation and reconstruction algorithms, i.e. SI signal regeneration, for an IEEE 802.11a/g standard with an FFT size $k=64$, a sample size $n=64$ and the CIR length $L=16$, defined by the $\mathrm{CP}$ in the standard. In the table, it can be seen that the most efficient TD-R approach with CC implementation, is roughly three times more expensive than the proposed FD-R

Table II. COMPUTATIONAL COMPLEXITY OF SI SignAL REGENERATION, CONSIDERING DIFFERENT ESTIMATION AND RECONSTRUCTION METHODS

\begin{tabular}{c|c|c|c|c}
\hline \hline \multirow{2}{*}{ Estimate } & \multirow{2}{*}{ Reconstruct } & \multicolumn{2}{|c|}{$\begin{array}{c}\text { Flop Count (addition } \\
+ \text { Multiplication) }\end{array}$} & For $N=1$ \\
\cline { 3 - 5 } & & Estimate & Reconstruct & Total Flops \\
\hline LS-TDE & Time [1], [12] & 8192 & $\begin{array}{c}10240 \cdot N,(\mathrm{DC}) \\
4272 \cdot N,(\mathrm{CC})\end{array}$ & $\begin{array}{c}18432,(\mathrm{DC}) \\
12464,(\mathrm{CC})\end{array}$ \\
\hline LS-TDE & Frequency & 8464 & $1680 \cdot N$ & 10144 \\
\hline LS-FDE & Time [2], [9] & 2592 & $\begin{array}{c}10240 \cdot N,(\mathrm{DC}) \\
4272 \cdot N,(\mathrm{CC})\end{array}$ & $\begin{array}{c}12832(\mathrm{DC}) \\
6864,(\mathrm{CC})\end{array}$ \\
\hline LS-FDE & Frequency & 1296 & $1680 \cdot N$ & 2976 \\
\hline
\end{tabular}

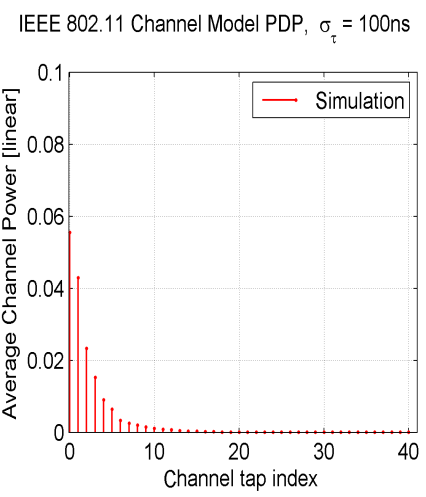

Frequency response of channel and averaged 100 OFDM symbols.

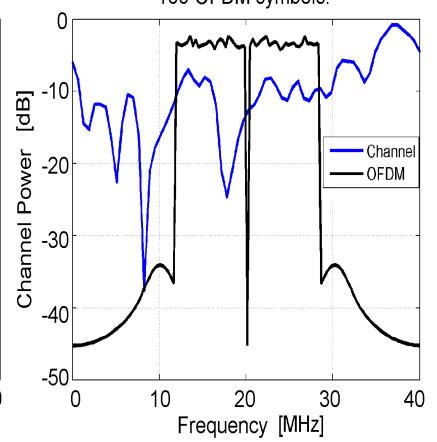

Figure 3. Channel PDP and frequency response for $\sigma_{\tau}=100 \mathrm{~ns}$ simulated using the IEEE 802.11 indoor channel model.

approach ( $\sim 61 \%$ reduction in flops). This clearly asserts FD$\mathrm{R}$ as a more efficient and much simpler approach over TD-R approach.

\section{PERformance Results}

In this section, we first explain the wireless channel model employed in our simulations, and then we present and discuss our results.

\section{A. Channel Model}

To analyze and compare the performance of our proposed FD-R approach in terms of achieved digital cancellation, we have simulated a time dispersive slowly fading conditions, incorporating the wireless channel effects on the transmitted OFDM packet. In our system, IEEE 802.11 indoor channel model proposed in [16] is used, which adopts the exponential model for generating the power delay profile (PDP), i.e.

$$
P(k)=\frac{1}{\sigma_{\tau}} \exp ^{-p T_{s} / \sigma_{\tau}} \quad p=0,1,2, \ldots, p_{\max },
$$

where $\sigma_{\tau}$ is the root mean square (RMS) delay spread, $T_{s}$ is the sampling time and $p$ is the discrete path index (taps), with $p_{\max }$ as the index of the last path (with smallest non-negligible power), and in [16] it is fixed as $p_{\max }=\left[10 \cdot \sigma_{\tau} / T_{S}\right]$. Now, assuming that the power of the $p^{\text {th }}$ channel tap has zero mean and variance of $\sigma_{p}^{2} / 2$, the coefficients of the impulse response are given as:

$$
h_{p}=\gamma_{p}+j \beta_{p}, \quad p=0,1,2, \ldots, p_{\max },
$$

where $\gamma_{p}$ and $\beta_{p}$ are statistically independent and identical Gaussian random variable each with $N\left(0, \sigma_{p}^{2} / 2\right)$, characterizing a multi path channel with $p_{\max }$ components. Fig. 3 presents a random realization of our simulated channel model for an RMS delay spread of 100 ns.

\section{B. Results}

The parameters setting of an OFDM symbol assumed in our simulations is given in Table I. One transmitted OFDM packet carries 100 OFDM symbols with 16-QAM modulation. The fading channel is simulated for 1000 random realizations with PDP considered for different RMS delays spreads. The simulated fading conditions are applied on the transmitted 


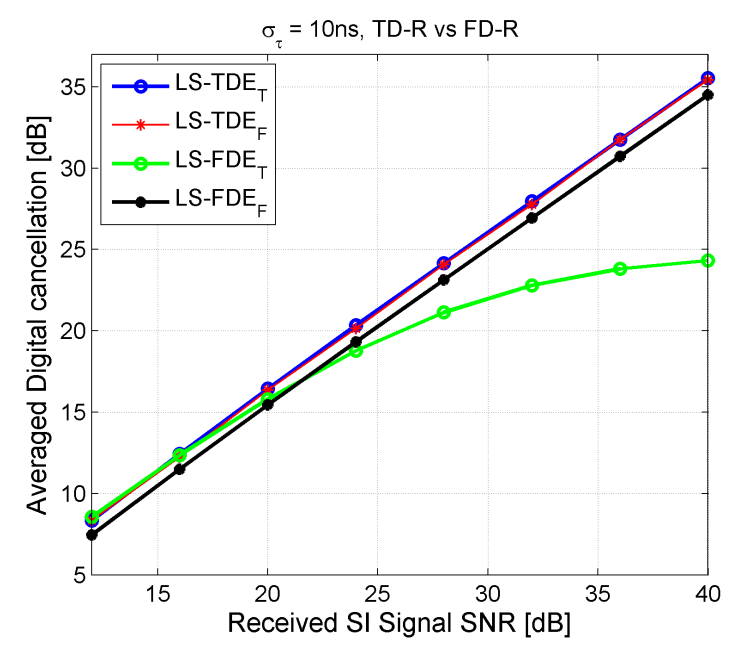

Figure 4. Performance of LS-TDE ${ }_{T}$ [1], [12], LS-FDE ${ }_{T}$ [2], [9] and proposed techniques under flat fading with $\sigma_{\tau}=10 \mathrm{~ns}$.

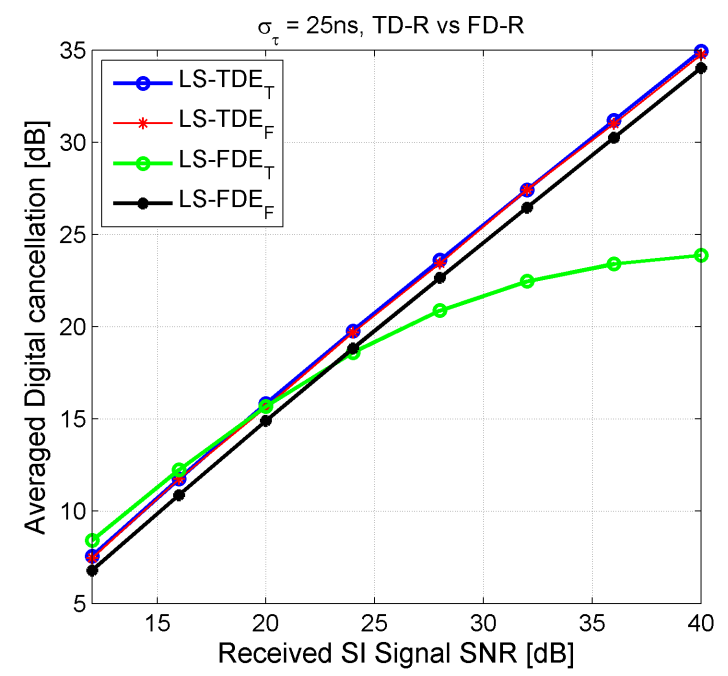

Figure 5. Performance of LS-TDE ${ }_{T}$ [1], [12], LS-FDE ${ }_{T}$ [2], [9] and proposed techniques under frequency selective fading with $\sigma_{\tau}=25 \mathrm{~ns}$.

OFDM packet, and their effects on the performance of the digital domain cancellation is observed. The legend in each figure indicates the estimation techniques, with subscripts denoting the reconstruction approach, i.e. 'T' is for TD-R and ' $\mathrm{F}$ ' is for FD-R.

First, we observed the digital cancellation performance of the proposed FD-R and existing TD-R approaches, with LSTDE and LS-FDE techniques against the received SNR of SI signal, under fixed RMS delay spreads. Fig. 4 presents the averaged digital cancellation performance under flat fading channels; whereas, Fig. 5 and Fig. 6 indicate the digital SI cancellation obtained in frequency selective channels with coherence bandwidth of roughly $8 \mathrm{MHz}$ and $2 \mathrm{MHz}$ (considering a correlation of 0.5 and above), respectively. These results show that a larger delay spread degrades the performance of all the employed SI cancellation techniques. Also, it can be noticed that the amount of digital cancellation increases with increasing SNR of the received SI signal, which is logical because with a higher SNR of an SI signal, a better estimate can

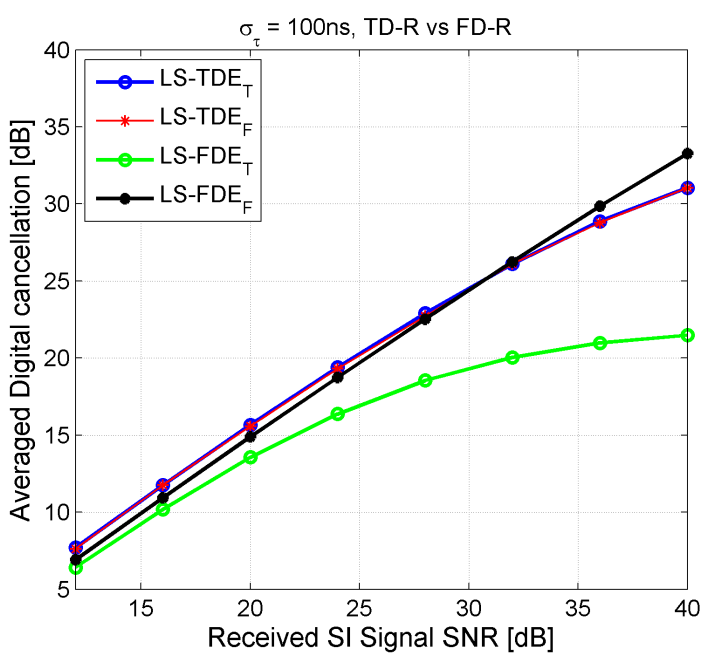

Figure 6. Performance of LS-TDE ${ }_{T}$ [1], [12], LS-FDE ${ }_{T}$ [2], [9] and proposed techniques under frequency selective fading with $\sigma_{\tau}=100 \mathrm{~ns}$.

be obtained. This further indicates the performance limitation of digital cancellation for low SNR SI signals. Additionally, it can be seen that the TD-R approach with LS-FDE technique (which is nearly seven times less expensive than LS-TDE technique) performs much poorer as compared to the rest of the SI suppression approaches. The main reason for this poor performance is that the LS-FDE techniques performs per carrier estimation, and taking the IFFT of the estimate distributes the concentrated power of channel taps to all $k$ points of the IFFT, which distorts the CIR estimate. This leads to poor reconstruction of the SI, which consequently reduces digital cancellation.

Secondly, we have investigated the digital cancellation performance of FD-R and TD-R approaches against increasing RMS delay spread. The plots shown in Fig. 7 and Fig. 8 present the digital cancellation performance with LS-TDE and LS-FDE techniques, in channels with fixed SNR of 30 $\mathrm{dB}$ and $40 \mathrm{~dB}$, respectively. In these results, the average achieved cancellation for both SNR levels is getting worse with increasing delay spread. Additionally, it can be noticed that for large RMS delay spread, the digital cancellation observed with LS-TDE $T$ and LS-TDE $F$, suffers far more than LS-FDE . $_{\text {. }}$ This is due to the fact that for delay spreads larger than the duration of guard interval, LS-TDE technique fails to capture the CIR efficiently, which eventually reduces the amount of digital cancellation; whereas, LS-FDE technique performs per carrier estimation, which makes it more resilient towards the selective nature of the channel.

A summary including the computational complexities and digital cancellation performances of different SI signal regeneration methods with $40 \mathrm{~dB}$ received SNR, is presented in Table III. These results clearly demonstrate the superiority of the proposed FD-R approach in terms computational requirements (a complexity reduction of nearly threefold), and digital cancellation performance, with a 5-7 dB more digital cancellation under extreme fading conditions. 


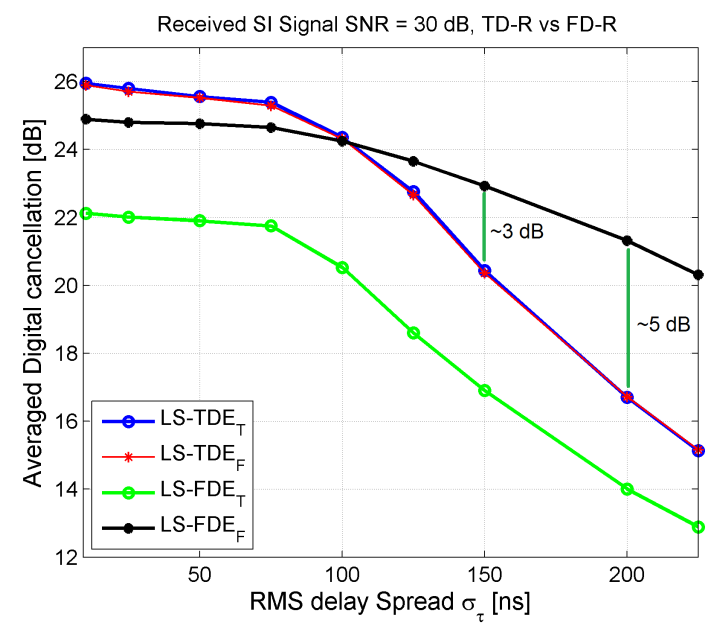

Figure 7. Performance of LS-TDE [1], [12], LS-TDE $_{T}$ [2], [9] and proposed techniques under time dispersive channel effects, and $30 \mathrm{~dB}$ received SNR of SI signal.

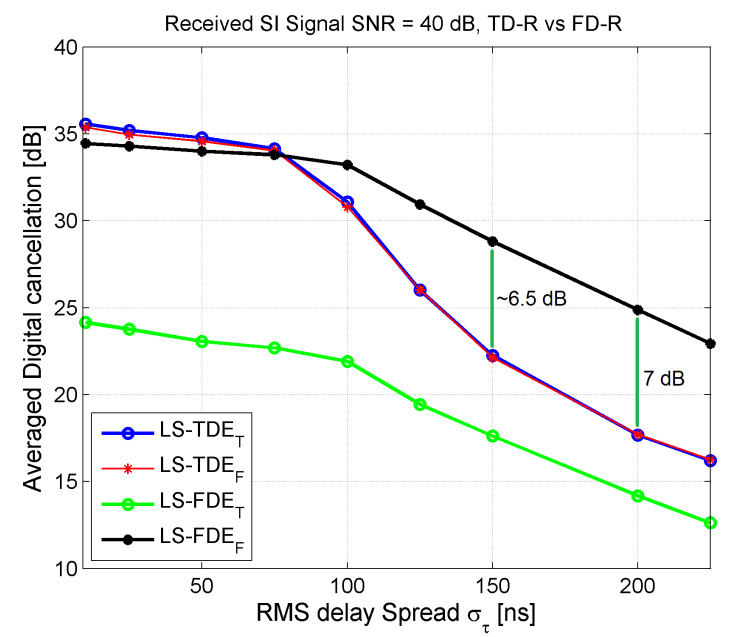

Figure 8. Performance of $\mathrm{LS}^{-\mathrm{TDE}_{T}}$ [1], [12], LS-TDE $T$ [2], [9] and proposed techniques under time dispersive channel effects, and $40 \mathrm{~dB}$ received SNR of SI signal.

\section{CONCLUSIONS}

Full-duplex is certainly a promising communication mode for wireless connectivity, due to the potential to significantly improve the spectral efficiency and throughput of a wireless network. In this work, a novel frequency domain based approach to reconstruct the SI signal in digital domain

Table III. COMPUTATIONAL COMPLEXITIES VS DIGITAL CANCELLATION PERFORMANCE SUMMARY

\begin{tabular}{|c|c|c|c|c|c|}
\hline \multirow[b]{2}{*}{$\begin{array}{l}\text { Digital } \\
\text { Cancellation } \\
\text { Technique }\end{array}$} & \multirow[b]{2}{*}{$\begin{array}{l}\text { Flop Delay Spread } \\
\text { count } \\
(\text { Est. + Rec.) }\end{array}$} & \multicolumn{4}{|c|}{$\begin{array}{l}\text { Digital Cancellation in [dB] } \\
\text { at } 40 \mathrm{~dB} \text { received SNR }\end{array}$} \\
\hline & & 25 & 75 & 100 & 200 \\
\hline LS-TDE $_{T}$ & $8192+4272 \cdot N,(\mathrm{CC})$ & 35.3 & 33.8 & 31.2 & 17.6 \\
\hline LS-TDE $F$ & $8464+1680 \cdot N$ & 35 & 33.6 & 31 & 17.6 \\
\hline $\mathrm{LS}_{-\mathrm{FDE}_{T}}$ & $2592+4272 \cdot N,(\mathrm{CC})$ & 23.8 & 22.5 & 22 & 14 \\
\hline LS-FDE $_{F}$ & $1296+1680 \cdot N$ & 34 & 33.4 & 33 & 25 \\
\hline
\end{tabular}

is presented. Via computational complexity evaluation, it is shown that the proposed approach is considerably efficient and reduces the computational requirements by $61 \%$. Also, our performance simulations, under time dispersive fading channel, have demonstrated that the performance of the proposed FD-R approach is not only comparable to TD-R, but its incorporation with LS-FDE has shown a 5-7 dB more digital SI suppression under highly selective fading channels. Nevertheless, the degradation of digital cancellation performance with large delay spreads does indicate the FD limitation for outdoor applications.

\section{REFERENCES}

[1] D. Bharadia, E. McMilin, and S. Katti, "Full duplex radios," $A C M$ SIGCOMM Computer Communication Review, vol. 43, no. 4, pp. 375386, 2013.

[2] M. Jain, J. I. Choi, T. Kim, D. Bharadia, S. Seth, K. Srinivasan, P. Levis, S. Katti, and P. Sinha, "Practical, real-time, full duplex wireless," in Proceedings of the 17th annual international conference on Mobile computing and networking, pp. 301-312, ACM, 2011.

[3] M. Duarte and A. Sabharwal, "Full-duplex wireless communications using off-the-shelf radios: Feasibility and first results," in 2010 Conference Record of the Forty Fourth Asilomar Conference on Signals, Systems and Computers, pp. 1558-1562, IEEE, 2010.

[4] E. Everett, A. Sahai, and A. Sabharwal, "Passive self-interference suppression for full-duplex infrastructure nodes," IEEE Transactions on Wireless Communications, vol. 13, no. 2, pp. 680-694, 2014.

[5] E. Ahmed, A. M. Eltawil, and A. Sabharwal, "Self-interference cancellation with nonlinear distortion suppression for full-duplex systems," in 2013 Asilomar Conference on Signals, Systems and Computers, pp. 1199-1203, IEEE, 2013.

[6] E. Ahmed, A. M. Eltawil, and A. Sabharwal, "Self-interference cancellation with phase noise induced ici suppression for full-duplex systems," in 2013 IEEE Global Communications Conference (GLOBECOM), pp. 3384-3388, IEEE, 2013.

[7] B. Radunovic, D. Gunawardena, P. Key, A. Proutiere, N. Singh, V. Balan, and G. Dejean, "Rethinking indoor wireless mesh design: Low power, low frequency, full-duplex," in Wireless Mesh Networks (WIMESH 2010), 2010 Fifth IEEE Workshop on, pp. 1-6, IEEE, 2010.

[8] E. Ahmed and A. M. Eltawil, "All-digital self-interference cancellation technique for full-duplex systems," IEEE Transactions on Wireless Communications, vol. 14, no. 7, pp. 3519-3532, 2015.

[9] M. Duarte, C. Dick, and A. Sabharwal, "Experiment-driven characterization of full-duplex wireless systems," IEEE Transactions on Wireless Communications, vol. 11, no. 12, pp. 4296-4307, 2012.

[10] A. Sahai, G. Patel, and A. Sabharwal, "Pushing the limits of full-duplex: Design and real-time implementation," arXiv preprint arXiv:1107.0607, 2011.

[11] Z. Zhang, K. Long, A. V. Vasilakos, and L. Hanzo, "Full-duplex wireless communications: Challenges, solutions, and future research directions," 2015.

[12] D. Bharadia and S. Katti, "Full duplex mimo radios," in 11th USENIX Symposium on Networked Systems Design and Implementation (NSDI 14), pp. 359-372, 2014.

[13] J. Terry and J. Heiskala, OFDM wireless LANs : a theoretical and practical guide. Indianapolis, Ind. Sams, 2002. OFDM : Orthogonal Frequency Division Multiplexing.

[14] D. L. Jones, "Decimation-in-time (dit) radix-2 fft," Connexions, September, vol. 15, p. 2006, 2006.

[15] R. Yavne, "An economical method for calculating the discrete fourier transform," in Proceedings of the December 9-11, 1968, fall joint computer conference, part I, pp. 115-125, ACM, 1968.

[16] N. Chayat, "Ieee 802.11-97/96," September, 1997. 